L'histoire du laser en médecine débute dès 1961, à peine

une année après l'invention

du premier laser. Le laser

en médecine peut agir selon

quatre types d'effets :

électromécanique, photoablatif, thermique et photochimique.

En 2010, les applications

médicales des lasers sont

très nombreuses.

Dans cet article, nous en

présentons deux, récemment développées : I'utilisation

du laser pour la cicatrisation cutanée et celle pour

le traitement du cancer

de la prostate.

\section{Applications médicales du laser}

Serge Mordon (serge.mordon@inserm.fr)

INSERM Unité 703, Université Lille Nord de France, CHRU, 59120 Loos
L'histoire du laser en médecine débute avec Campbell en ophtalmologie (1961) et Goldman en dermatologie (1963). Le laser à dioxyde de carbone $\left(\mathrm{CO}_{2}\right)$, introduit par Polanyi et Kaplan à partir de 1965, fut tout d'abord proposé aux chirurgiens avec le concept d'un bistouri "optique ». Le recours aux fibres optiques dans le courant des années 1970 a ouvert le champ des applications lasers à l'endocavitaire, grâce à la possibilité d'introduire la fibre dans le canal opérateur d'un endoscope. Depuis le début des années 1980, les applications du laser se sont particulièrement développées. En 2010, l'ASLMS (American Society for Laser in Medicine andSurgery) etlaSFLM(SociétéFrancophone des Lasers Médicaux) fêtent leurs trente années d'existence. Les applications médicales sont aujourd'hui très nombreuses, et il n'est pas possible en quelques pages d'en faire un panorama complet.

Cet article se limitera donc à introduire les mécanismes d'action des lasers en médecine, puis à évoquer deux applications en développement qui concernent des pathologies très fréquentes.

\section{Mécanismes d’action du laser}

Il est possible de proposer une classification de l'interaction laser-tissu biologique selon quatre types d'effets [1]. Cette distinction dépend du temps d'exposition et, donc, de l'irradiance appliquée (fig. 1).

- L'effet électromécanique (action disruptive), qui est obtenu avec des impulsions de 10 ps à 10 ns et des irradiances de l'ordre de $10^{9}$ à $10^{12} \mathrm{~W} / \mathrm{cm}^{2}$.

- L'effet photoablatif, qui est obtenu avec des impulsions de 10 ns à 100 ns. Dans ce cas, plus que l'irradiance, c'est le domaine spectral qui est important, l'effet photoablatif nécessitant des photons énergétiques (UV).

- L'effet thermique, qui est obtenu avec des impulsions de $1 \mathrm{~ms}$ à quelques secondes et des irradiances de l'ordre $10^{1}$ à $10^{6} \mathrm{~W} / \mathrm{cm}^{2}$.

- L'effet photochimique est obtenu uniquement en combinaison avec un photosensibilisant, avec des durées d'illumination s'étendant de la dizaine de secondes à la dizaine de minutes, et des irradiances généralement très faibles.

>>

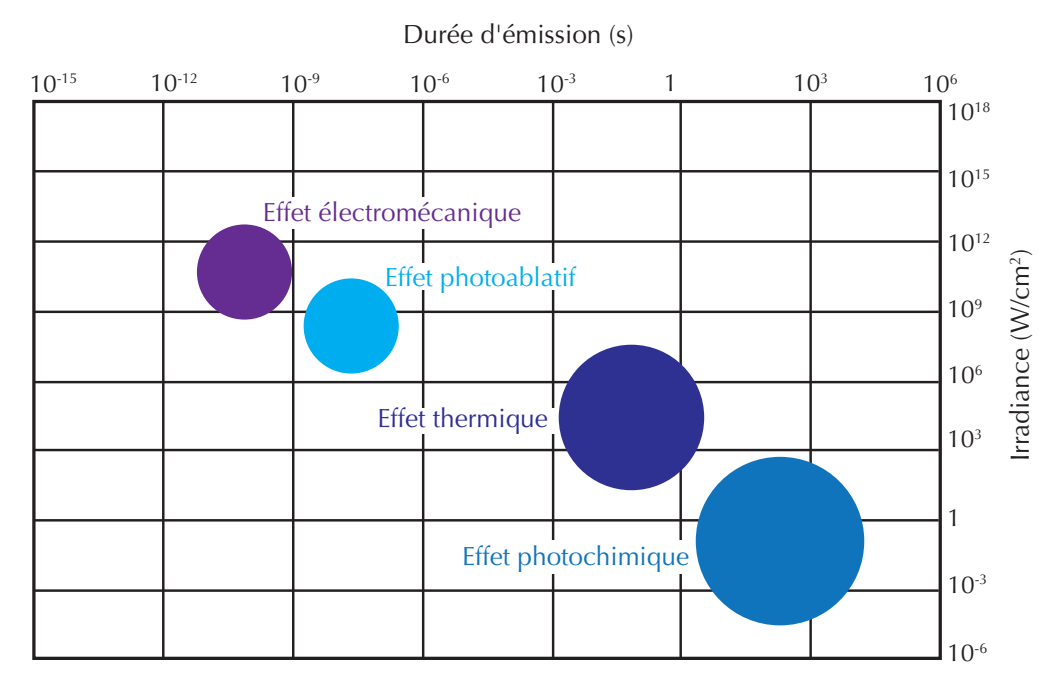

1. Les différents effets obtenus avec les lasers sur les tissus biologiques, en fonction de la durée d'émission du laser et de l'irradiance (S. Mordon, 2010.) 


\section{L'effet électromécanique}

Lorsqu'une impulsion laser très courte (nanoseconde et en dessous) est focalisée sur une cible tissulaire, créant ainsi des irradiances élevées (de l'ordre de $10^{9}$ à $10^{12} \mathrm{~W} / \mathrm{cm}^{2}$ ), il est possible d'obtenir localement des champs électriques importants $\left(10^{6}\right.$ à $\left.10^{7} \mathrm{~V} / \mathrm{m}\right)$, comparables aux champs atomiques ou intramoléculaires. De tels champs induisent un claquage électrique du matériau de la cible, ayant pour résultat la formation d'un plasma. L'onde de choc associée à l'expansion du plasma engendre des ondes de pression extrêmement importantes et, par conséquent, une rupture mécanique de la structure tissulaire. Cet effet électromécanique est généralement obtenu avec des lasers $\mathrm{Nd}: \mathrm{YAG}$, fonctionnant en mode déclenché (ns) ou en mode bloqué (ps). En médecine, cet effet est utilisé en ophtalmologie pour détruire des membranules de l'œil qui surviennent souvent après l'implantation d'un cristallin artificiel. En dermatologie, on a recours à des lasers fonctionnant en mode déclenché (rubis, alexandrite, $\mathrm{Nd}: \mathrm{YAG}$ ) pour le traitement de lésions pigmentées ou le détatouage. Dans ce dernier cas, le choix de la longueur d'onde dépend de la couleur des encres de tatouage afin d'obtenir une action spécifique.

\section{L'effet photoablatif}

L'effet photoablatif, appelé aussi photodécomposition ablative, est basé sur l'utilisation de photons présentant une énergie supérieure à l'énergie de liaison des molécules biologiques. En effet, des photons ayant une énergie de l'ordre de $4 \mathrm{eV}$ à $6 \mathrm{eV}$ sont susceptibles de dissocier des liaisons peptidiques ou les liaisons carbone-carbone des chaînes polypeptidiques. Le processus photoablatif consiste ainsi en une dissociation ou une rupture de la matière et en l'expulsion des fragments à une vitesse supersonique. Les lasers émettant dans l'ultraviolet, tels que les lasers à excimères (ArF: 193 nm-6,4 eV ou $\mathrm{XeCl}: 308 \mathrm{~nm}-4 \mathrm{eV}$ ) ou bien un laser $\mathrm{Nd}$ :YAG quadruplé en fréquence (266 nm-4,7 eV), sont bien adaptés à la photoablation. Cet effet est particulièrement utilisé en ophtalmologie pour la chirurgie réfractive de la cornée, qui consiste à en modifier la courbure. Il est ainsi possible de corriger des myopies, des astigmatismes légers à modérés, ainsi que des petites hypermétropies.

\section{L'effet thermique}

L'action thermique constitue aujourd'hui le mécanisme prédominant des applications thérapeutiques des lasers. L'effet thermique des lasers est un processus complexe comprenant trois phénomènes : une conversion de la lumière laser en chaleur, un transfert de chaleur dans le tissu et une réaction tissulaire dépendante de la température. En fonction de la durée du chauffage réalisé et de l'élévation de la température du tissu, on peut obtenir une hyperthermie (élévation de température supérieure à $37{ }^{\circ} \mathrm{C}$ ne conduisant pas à une mort cellulaire), ou une coagulation (nécrose irréversible sans destruction tissulaire immédiate), ou une volatilisation d'un volume tissulaire donné. Cet effet est utilisé dans la plupart des applications médicales des lasers, et tout particulièrement en ophtalmologie (décollement de la rétine), dermatologie (destruction de lésions cutanées), chirurgie, etc.

\section{L'effet photochimique}

L'effet photochimique, plus souvent nommé Photothérapie Dynamique (Photodynamic Therapy: PDT), consiste à sensibiliser électivement une lésion par l'administration d'un photosensibilisant, puis à la détruire par une activation lumineuse spécifique de celui-ci. C'est une démarche en deux temps :

i) l'application ou l'administration d'un photosensibilisant qui s'accumule de manière variable, dans un délai de quelques heures à trois jours, dans la lésion à traiter ; ii) l'éclairage de la lésion par une lumière de faible intensité, sans effet thermique, dont la longueur d'onde est préférentiellement absorbée par le photosensibilisant.

L'excitation de ce dernier initie alors par transfert d'énergie une cascade de réactions finalement cytotoxiques, qui peuvent être de deux types : dans les mécanismes de type I, le photosensibilisant va réagir chimiquement avec des molécules en interaction directe. Des réactions d'oxydoréduction conduisent à des espèces radicalaires et, par des processus complexes faisant généralement intervenir l'oxygène, à la dégradation des molécules avoisinantes. Le photosensibilisant est le plus souvent détruit au cours de ces réactions. Dans les mécanismes de type II, un transfert d'énergie à l'oxygène amène celui-ci à l'état singulet, ${ }^{1} \mathrm{O}_{2}$. Le photosensibilisant revient à son état fondamental et il est prêt à une nouvelle collecte d'énergie lumineuse. L'oxygène singulet est une espèce oxydante. Les acides aminés, certaines bases nucléiques et, à un moindre degré, les chaînes lipidiques composant les membranes sont particulièrement sensibles à son action. Les mécanismes de type II sont prépondérants dans le processus photochimique. Le stress oxydatif pourrait être relayé par un mécanisme d'apoptose ${ }^{(1)}$, agissant à la fois sur les membranes cellulaires et sur la mitochondrie.

La présence d'un photosensibilisant est requise pour l'obtention d'un effet photochimique. Un photosensibilisant est une molécule non toxique pour l'organisme et dénuée d'activité thérapeutique propre. Il est idéalement caractérisé par : i) une fixation ou une rétention élective par les cellules cancéreuses ; ii) par un (ou parfois plusieurs) pics d'absorption lumineuse. Aujourd'hui, plusieurs photosensibilisants disposent de l'AMM (Autorisation de Mise sur le Marché) et d'autres sont en cours d'évaluation.

\section{Le laser pour l'aide à la cicatrisation cutanée}

Plus de soixante-dix millions d'interventions chirurgicales sont pratiquées chaque année aux États-Unis, la majorité d'entre elles impliquant une incision cutanée et donc une cicatrice. Pratiquement tous les individus auront dans leur vie une ou plusieurs interventions chirurgicales entraînant des cicatrices. Si, dans la majorité des cas, la cicatrice résultante est discrète, il n'est pas rare de voir se développer des cicatrices hypertrophiques ou chéloïdes ${ }^{(2)}$.

Les patients et les médecins sont ainsi motivés pour améliorer le résultat esthétique des cicatrices. Contrairement à la salamandre, les mammifères ont une capacité très limitée de régénération d'organes ou de cellules. Toute incision de la peau conduit à une cicatrice qui semble être le résultat de la rapide interposition de la fibrose (accumulation anormale de macromolécules de la matrice extracellulaire telles que le collagène, l'élastine...) des tissus, ce qui empêche la régénération tissulaire ultérieure. Cependant, la chirurgie in utero a pu montrer qu'une plaie peut se refermer sans traces visibles.

Récemment, différentes équipes ont cherché à bloquer la formation de cicatrices, en particulier en manipulant la production de certains facteurs de croissance, qui 
agissent sur les cellules à l'origine de la réparation tissulaire (fibroblastes en particulier) [2]. Bien que d'énormes progrès aient été accomplis dans la délimitation de la myriade de facteurs impliqués dans des conditions normales et pathologiques de la réparation des tissus, ces résultats n'ont pas abouti à des avancées substantielles dans les soins aux patients. Il est devenu clair qu'un agent chimique seul, tel que l'administration d'un facteur de croissance, n'a qu'un impact modéré sur la réparation des plaies en milieu clinique, le plus probablement en raison de la plasticité et de la redondance des composants des processus de réparation de la blessure, ou en raison de sa dégradation rapide dans la plaie. Cependant, de récents résultats suggèrent que des changements de l'environnement physique (thermique ou mécanique) peuvent modifier le processus de cicatrisation. Des traitements, au moyen de lasers thermiques, ont clairement montré qu'il était possible d'obtenir un quasi-processus de régénération de la plaie [3].

Depuis les premiers travaux sur l'amélioration de la cicatrisation cutanée par laser, qui datent d'une trentaine d'années, avec Abergel qui utilisa un laser $\mathrm{Nd}$ :YAG à $1,06 \mu \mathrm{m}$, de récentes études cliniques ont démontré que les lasers pourraient permettre de modifier la réponse inflammatoire et, par conséquent, le processus cicatriciel. À ce jour, deux lasers différents ont été utilisés : le laser à colorant pulsé qui émet à $585 \mathrm{~nm}$ (mais son action est trop superficielle) et la diode laser qui émet à $810 \mathrm{~nm}$ (technique LASH). En effet, c'est dans la zone qui s'étend entre 650 et
$1200 \mathrm{~nm}$ que l'absorption par l'eau est la plus faible et sera donc la plus propice à une illumination en profondeur (fig. 2).

La technique LASH a été mise au point à Lille. Les premiers travaux expérimentaux ont été réalisés en 2000 sur le rat Hairless ; ils ont montré que des sutures chirurgicales, traitées par une diode laser à $810 \mathrm{~nm}$, donnaient des cicatrices nettement moins visibles que les contrôles (voire totalement inexistantes) [4]. Une analyse histologique a confirmé l'accélération du processus cicatriciel et une meilleure résistance à la rupture des plaies traitées par laser, par rapport aux contrôles.

La technique LASH est maintenant mise en œuvre au moyen d'un système portatif à diode laser à 810 nm (Ekkylite, Ekkyo, Aix-en-Provence), qui agit par effet thermique (fig. 3a). Ce laser n'est utilisé qu'à la fin de l'intervention chirurgicale, immédiatement après la fermeture de l'incision opératoire. Il délivre un spot rectangulaire de $20 \times 4 \mathrm{~mm}^{2}$. Ce spot est déplacé par l'opérateur afin de traiter la totalité de l'incision (fig. 3b). Ainsi, une incision de $20 \mathrm{~cm}$ nécessite une durée de traitement de l'ordre de deux minutes.

Cette approche originale de cicatrisation cutanée, assistée par une diode laser de $810 \mathrm{~nm}$, a fait l'objet d'évaluations cliniques. Par exemple, sur une série de trente patients évalués à douze mois, une nette amélioration de la cicatrice traitée au laser est observée (fig. 4). Une analyse d'empreintes en silicone des cicatrices montre une diminution moyenne de hauteur de 38,1\% à douze mois pour les patients traités au laser comparés au groupe témoin. Cette étude montre aussi

>>

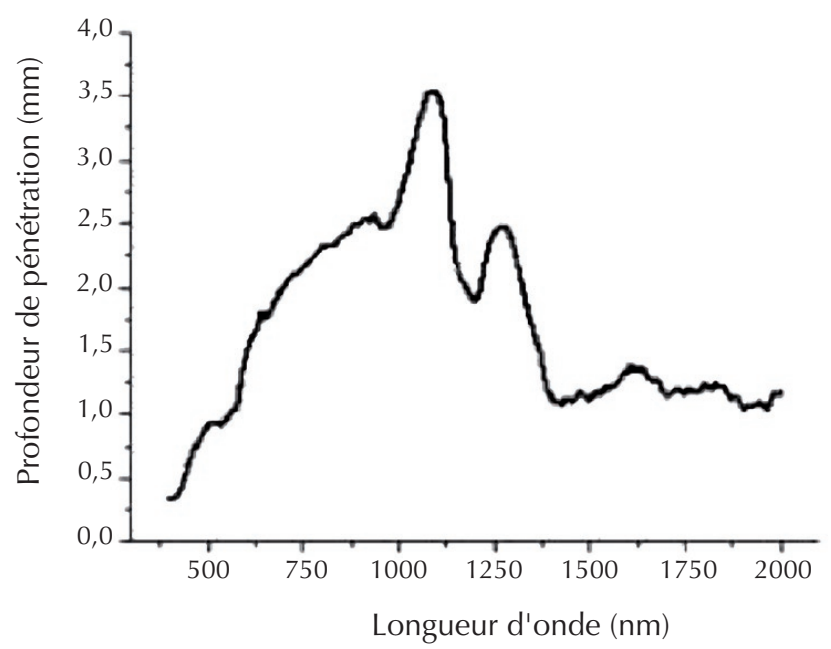

2. Profondeur de pénétration d'un rayonnement laser dans la peau, en fonction de la longueur d'onde.

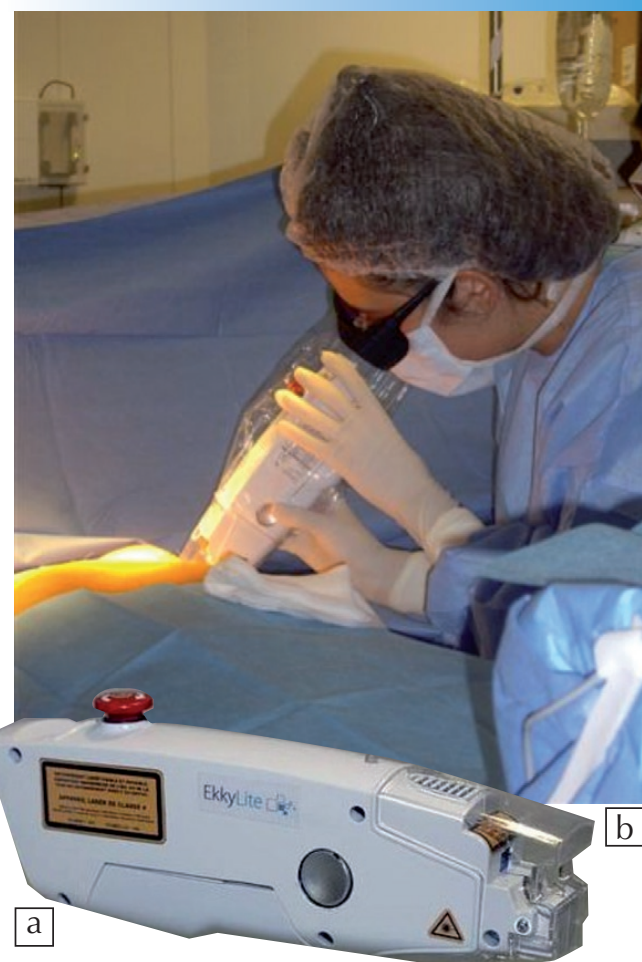

3. (a) Laser portatif développé par la société Ekkyo (Aix-en-Provence, France). Ce laser émet un faisceau de $4 \mathrm{~mm} \times 20 \mathrm{~mm}$. (b) Le faisceau est appliqué le long de l'incision immédiatement après fermeture. Les paramètres typiquement utilisés sont une longueur d'onde de $810 \mathrm{~nm}$, une puissance de $8 \mathrm{~W}$ et une fluence de 80 à $130 \mathrm{~J} / \mathrm{cm}^{2}$
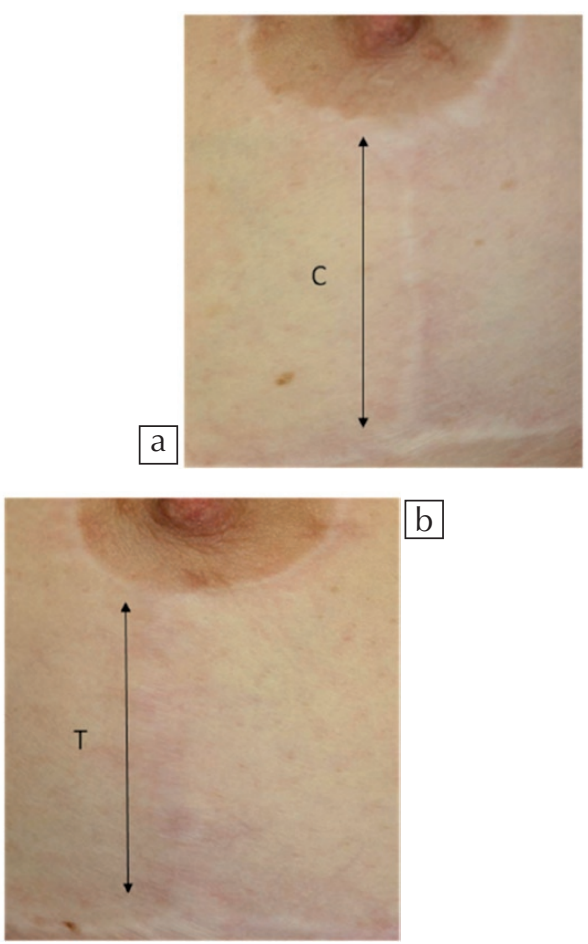

4. Traitement par diode laser à $810 \mathrm{~nm}$ (technique LASH) d'une cicatrice verticale au sein chez une femme de 35 ans. (a) Sein droit non traité. (b) Sein gauche traité par laser (résultat à douze mois). Les parties traitées (T) et contrôlées (C) sont indiquées. Le laser a été appliqué le long de l'incision immédiatement après suture (T), avec une fluence de $110 \mathrm{~J} / \mathrm{cm}^{2}$, et a conduit à une quasi-disparition de la cicatrice. 


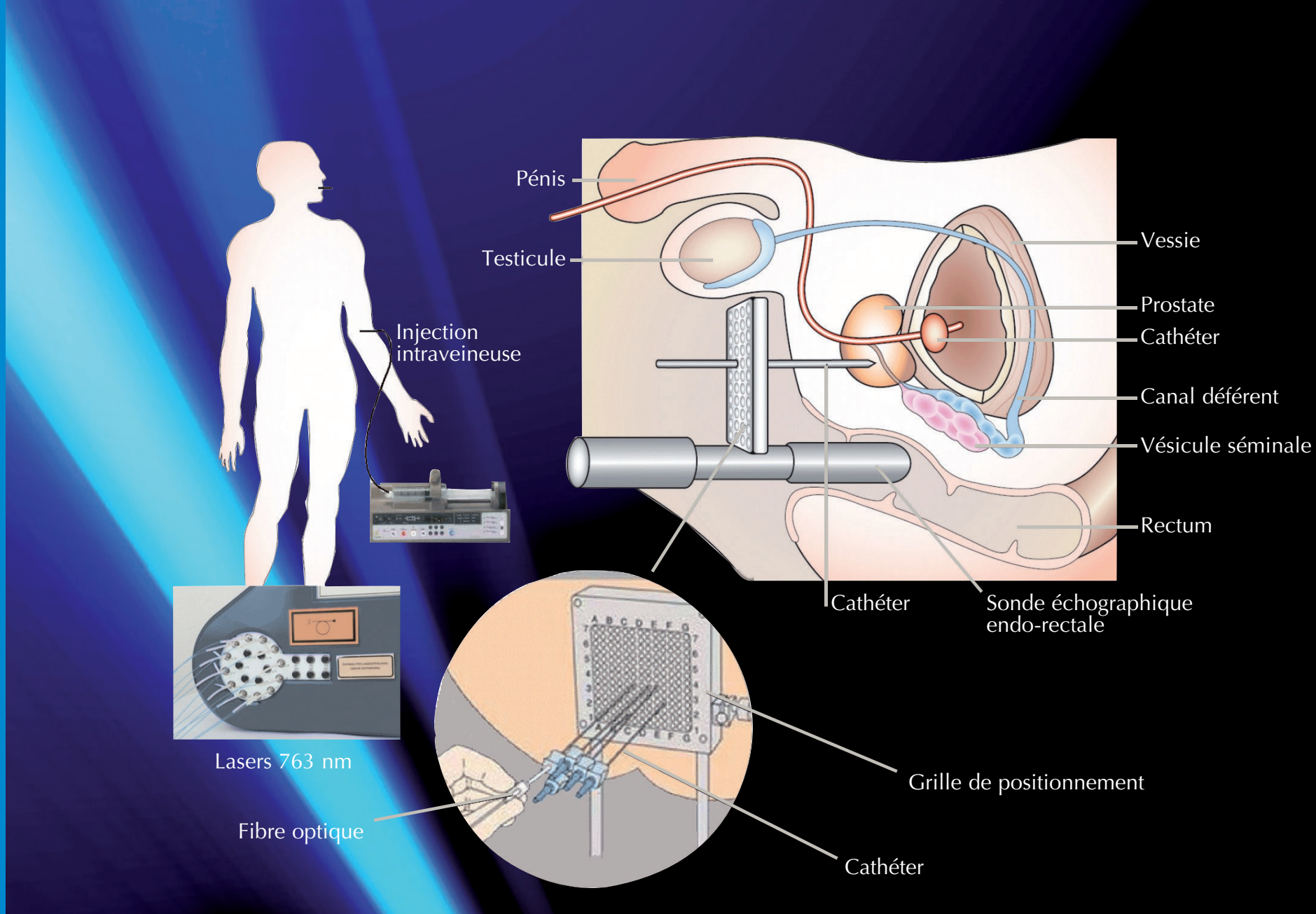

5. Schéma illustrant le principe du traitement par PDT du cancer de la prostate. Les fibres sont introduites dans des cathéters positionnés dans la prostate. Leur position est contrôlée par échographie endorectale. Le photosensibilisant (Tookad - 4 mg/kg) est injecté par voie intraveineuse. L'illumination des fibres est faite au moyen d'un laser de longueur d'onde $763 \mathrm{~nm}$, pendant une durée de vingt-deux minutes. 
le rôle déterminant de la dose $\left(\mathrm{J} / \mathrm{cm}^{2}\right)$ appliquée. Pour les huit patients traités à faible dose $\left(<80 \mathrm{~J} / \mathrm{cm}^{2}\right)$, il n'y avait pas de différence significative dans le segment des patients traités par rapport au segment de contrôle. En revanche, pour les patients traités avec des doses supérieures à $115 \mathrm{~J} / \mathrm{cm}^{2}$, il existe un risque de brûlures superficielles [5]. Heureusement, grâce à un spot laser parfaitement homogène, le laser Ekkyo assure une excellente reproductibilité des paramètres de tir. Récemment, une autre étude clinique a pu montrer que la technique LASH pourrait jouer un rôle déterminant dans la prévention des cicatrices hypertrophiques [6].

\section{Le laser pour le traitement du cancer de la prostate}

Le cancer de la prostate est le premier cancer masculin. En France, il atteint un homme sur huit avant l'âge de 75 ans, avec plus de 60000 nouveaux cas et 10000 décès par an. Compte tenu de l'augmentation régulière de l'espérance de vie, c'est un cancer en progression très rapide. Aujourd'hui, les cancers de la prostate sont diagnostiqués de plus en plus tôt, et donc découverts à un stade localisé présentant des chances de guérison accrues. On propose généralement un traitement curatif à tout patient ayant une maladie localisée, c'est-à-dire se limitant à la glande prostatique, et dont l'espérance de vie est supérieure à dix ans. Jusqu'à présent, il existe trois types de traitements curatifs pour les cancers localisés : la chirurgie avec ablation complète de la prostate, la radiothérapie et la curiethérapie. Ces traitements présentent un caractère relativement invasif et des effets secondaires pénibles (incontinence urinaire, dysfonction sexuelle...).

En dehors de ces trois traitements, de nouvelles méthodes thérapeutiques, qualifiées de "mini invasives", sont en cours d'évaluation. En particulier, la photothérapie dynamique (PDT) interstitielle de la prostate laisse entrevoir la possibilité d'une technique peu invasive, peu douloureuse et aux effets secondaires moindres. La PDT des cancers localisés de la prostate est aujourd'hui principalement réalisée avec la padéliporfine $\left(\right.$ Tookad $^{\circledR}$ Soluble - société Steba-Biotech). Cette substance, développée avec l'Institut Weizmann, est une forme modifiée par synthèse chimique de la chlorophylle. Elle est injectée par voie intraveineuse et son effet est activé par exposition à une lumière de longueur d'onde spécifique $753 \mathrm{~nm}$. Cette longueur d'onde permet une bonne pénétration de la lumière dans la prostate. L'association de la lumière laser et du produit photosensibilisant provoque une destruction du tissu prostatique en l'espace de quelques jours, essentiellement grâce à l'action sur le réseau vasculaire. Le tissu prostatique n'étant plus vascularisé, les cellules cancéreuses se situant dans la prostate sont elles-mêmes détruites [7].

En pratique, sur la base des images obtenues en IRM qui permettent de connaître la position des cancers localisés, un logiciel de planification définit les positions des fibres optiques qui sont introduites dans la prostate par voie périnéale au sein des lésions cancéreuses. Ces fibres sont mises en place à travers une aiguille introduite via le périnée dans la prostate sous contrôle échographique. Elles sont ensuite connectées à un laser qui permet de réaliser l'excitation lumineuse de la substance préalablement injectée par voie intraveineuse, pendant une durée de vingt-deux minutes qui correspond au temps de demi-vie de la padéliporfine dans le réseau vasculaire (fig. 5). Cette technique permet une destruction très sélective de zones localisées de la prostate, comme l'illustre la figure 6. Le traitement est bien supporté par le patient, qui arrive à l'hôpital le matin et peut rentrer chez lui quelques heures après la séance de PDT [8].

\section{Conclusion}

Ces deux exemples illustrent des applications très récentes des lasers. Les progrès dans la miniaturisation des lasers laissent entrevoir une multiplication de leurs applications médicales. En effet, le fort encombrement, le coût important, la difficulté de mise en œuvre des lasers médicaux, ont souvent freiné leur dissémination dans les blocs chirurgicaux. Parallèlement, grâce aux progrès de l'imagerie, il devient possible de détecter des cancers à un stade précoce. La voie interstitielle (la fibre est insérée via une canule de très petit diamètre) permet d'introduire la fibre de traitement au sein de la lésion, souvent sous anesthésie locale. La procédure chirurgicale est donc beaucoup moins agressive pour le patient, avec des temps d'hospitalisation considérablement réduits.

(1) Apoptose : mort cellulaire programmée.

(2) Chéloïde : se dit d'une cicatrice en bourrelet, douloureuse au toucher.

\section{Références}

1 - S. Mordon et C. Boccara, "Applications médicales ", dans Le laser, coordonné par N. Treps et F. Bretenaker, Collection " Une introduction à... ", EDP Sciences, 2010.

2 • M. Shah,D.M. Foreman,M.W. Ferguson, “Neutralisation of TGF-beta 1 and TGF-beta 2 or exogenous addition of TGF-beta 3 to cutaneous rat wounds reduces scarring", J Cell. Sci. 108 (1995) 985-1002.

3 - S. Mordon et al., « Lasers thermiques et cicatrisation cutanée », Med. Sci (Paris) 26 (2010) 89-94.

4 A. Capon et al., "Laser assisted skin closure (LASC) by using a 815-nm diode-laser system accelerates and improves wound healing", Lasers Surg. Med. 28 (2001) 168-175.

5 - A. Capon et al., "Scar Prevention Using Laser-Assisted Skin Healing (LASH) in Plastic Surgery. Aesthetic", Plast. Surg. (2010) Jan 28.

6 A. Capon, G. larmarcovai et S. Mordon, "Laserassisted skin healing (LASH) in hypertrophic scar revision", J. Cosmet. Laser Ther. 11 (2009) 220-223.

7 • S.R. Davidson et al., "Treatment planning and dose analysis for interstitial photodynamic therapy of prostate cancer", Phys. Med. Biol. 54 (2009) 2293-2313.

8 • N. Arumainayagam et al., "Photodynamic therapy for focal ablation of the prostate", World J. Urol. (2010) May 9. 